\title{
Amorphous Cobalt Boron Alloy@Graphene Oxide Nanocomposites for Pseudocapacitor Applications
}

Wei Zhang ${ }^{1}$, Xiaoli $\mathrm{Du}^{2}$, Yueyue Tan ${ }^{1}$, Jinbo $\mathrm{Hu}^{3}$, Zhen $\mathrm{Li}^{1}$, Bohejin Tang ${ }^{1,3,{ }^{*}}$

${ }^{1}$ College of Chemistry and Chemical Engineering, Shanghai University of Engineering Science, Shanghai 201620, China

${ }^{2}$ School of Materials Engineering, Shanghai University of Engineering Science, Shanghai 201620, China

${ }^{3}$ Department of Chemistry,University of Minnesota, 207 Pleasant St. SE, Minneapolis, Minnesota 55 455, United States

[Received in 15 November 2015; Received in revised form 11 April; Accepted 16 May 2016]

*Corresponding author.

E-mail address: tangbohejin@sues.edu.cn (Bohejin Tang).

Amorphous Co-B alloy nanoparticles grown on graphene sheets were synthesized via a chemical reduction approach and successfully used for an application as a pseudocapacitor. This study aims to improve the capacity and cycling stability of amorphous Co-B alloy nanoparticles grown on conductive graphene sheets. The products were characterized by X-ray powder diffraction, scanning electron microscopy and transmission electron microscopy. As electrode materials for pseudocapacitors, the amorphous Co-B alloy grown on graphene oxide (Co-B@GO) exhibits a high specific capacitance of $460 \mathrm{~F} \mathrm{~g}^{-1}$, which is nearly 1.5 times greater than that of bare $\mathrm{Co}-\mathrm{B}$ nanoparticles at $1 \mathrm{~A} \mathrm{~g}^{-1}$. The specific capacitance preserved $84 \%$ of the initial capacitance even after 1000 cycles at a scan rate of $10 \mathrm{~m} \mathrm{~V}^{-1}$, suggesting its promising potential as pseudocapacitor materials.

Key words: Pseudocapacitor, Electrode, Cobalt-boron alloy, Amorphous alloy, Graphene oxide

\section{Introduction}

Electrochemical capacitors (so-called supercapacitors) have become one of the most promising candidates for next-generation power devices, with desirable properties of high power density, fast 
charging rate and excellent cycling stability ${ }^{[1,2]}$. Based on the charge storage mechanism, supercapacitors are classified as electrochemical double layer capacitors (EDLCs) with physical adsorption of ions at the interface of the electrode surface and the electrolyte, and pseudocapacitors with a fast reversible faradaic charge transfer at the electrode surface ${ }^{[3]}$. Currently, most commercial supercapacitors are EDLCs that are based on carbon materials ${ }^{[4,5]}$. However, the low energy density of these devices has so far barred them from many important applications ${ }^{[6]}$. Transition metal oxides such as $\mathrm{MnO}_{2}{ }^{[7]}, \mathrm{CuO}^{[8]}, \mathrm{ZrO}_{2}{ }^{[9]}$, and $\mathrm{SnO}_{2}{ }^{[10]}$, are a group of very promising pseudocapacitor electrode materials to replace expensive and toxic $\mathrm{RuO}_{2}$. Although they exhibit higher specific capacitance and energy density than EDLCs, they usually suffer from poor cyclability due to irreversible redox reactions at the surface of active materials ${ }^{[11]}$.

A recently adopted approach to overcome these issues is to develop composites of carbon-based materials and active materials as hybrid electrodes, which combines advantages of EDLCs and pseudocapacitors $^{[8,12]}$. In the carbon materials, graphene is an ideal matrix with many fascinating properties, such as extraordinarily high electrical conductivity, large specific surface area, high mechanical flexibility, and pronounced thermal and chemical stability ${ }^{[13,14]}$. Therefore, much research has focused on the fabrication of composites based on graphene for pseudocapacitor applications, generating special properties by the reinforcement or modification of each other. For example, Sahu et al. ${ }^{[15]}$ reported the growth of interconnected $\mathrm{ZnO}$ nanorings initiated through functionalities present in the edge planes of lacey graphene nanoribbons (GNRs). The test results show that a $\mathrm{ZnO} / \mathrm{GNR}$ nanocomposite electrode exhibits a $2.0 \mathrm{~V}$ potential window in an aqueous electrolyte and an ultra-short time constant $(0.08 \mathrm{~s})$ in an asymmetric supercapacitor device with lacey reduced graphene oxide nanoribbons (LRGONRs) as a negative electrode. Furthermore, the $\mathrm{ZnO/GNR} \mathrm{nanocomposite} \mathrm{electrode} \mathrm{exhibits} \mathrm{high} \mathrm{capacitance} \mathrm{and} \mathrm{cycling} \mathrm{stability} \mathrm{as} \mathrm{compared} \mathrm{with}$ $\mathrm{ZnO}$ synthesized by the same procedure without the support LGONR.

Compounds of cobalt exhibit exceptional physio-chemical properties and have been widely applied in fabricating pseudocapacitor electrode ${ }^{[16,17]}$. For instances, Mai et al. ${ }^{[18]}$ synthesized the three-dimensional (3D) multicomponent oxide, $\mathrm{MnMoO}_{4} / \mathrm{CoMoO}_{4}$. They fabricated asymmetric supercapacitors based on hierarchical $\mathrm{MnMoO}_{4} / \mathrm{CoMoO}_{4}$ heterostructured nanowires, which show a specific capacitance of $187.1 \mathrm{~F} \mathrm{~g} \mathrm{~g}^{-1}$ at a current density of $1 \mathrm{~A} \mathrm{~g}^{-1}$, and good reversibility with a cycling efficiency of $98 \%$ after 1000 cycles. Tian et al. ${ }^{[19]}$ established a simple and efficient strategy 
to achieve both ultrahigh energy and power densities of micropseudocapacitors (MPCs) with the plantation of pillar arrays in the microspirals with ultrathin nanosheets. A compound of cobalt as the microelectrode delivers both ultrahigh energy density of about $34.9 \mathrm{mWh} \mathrm{cm}^{-3}$ at a scan rate of 10 $\mathrm{mV} \mathrm{s}^{-1}$ and high power density of $193.4 \mathrm{~W} \mathrm{~cm}^{-3}$ at the ultrahigh scan rate of $200 \mathrm{~V} \mathrm{~s}^{-1}$. In addition, it also demonstrates an excellent cycling stability. However, researchers seldom attempt to fabricate $\mathrm{Co}_{x} \mathrm{~B}_{y}$ as electrode materials for pseudocapacitor application. Liu et al. ${ }^{[20]}$ reported that a series of Co-B alloys were synthesized successfully by arc melting, resulting in good cycling stability. However, this approach is not suitable for mass production due to their high synthesis costs as well as present technological difficulties. Wang et al. ${ }^{[21]}$ synthesized ultrafine amorphous alloy powders of Co-B and tested them for possible application as electroactive anodic materials. The results demonstrated that the Co-B particles exhibit excellent electrochemical reversibility and a high charge-discharge capacity. Moreover, we ${ }^{[22]}$ reported that mesoporous carbon electrodes can be doped with Co-B nanoparticles via a chemical reduction approach and successfully used for pseudocapacitor application, which presents a maximum specific capacitance of $531 \mathrm{~F} \mathrm{~g}^{-1}$ at a scanning rate of $5 \mathrm{mV} \mathrm{s}^{-1}$. However, the cycling performance of amorphous $\mathrm{Co}-\mathrm{B}$ alloy has limited its commercial attractiveness for pseudocapacitor applications.

In this study, we prepared nanocomposites of Co-B particles grown on graphene support, based on an oxidation-reduction reaction between graphene oxide and $\mathrm{CoCl}_{2}$. The electrochemical properties of the Co-B@GO nanocomposites as pseudocapacitor electrodes were investigated by a series of electrochemical tests.

\section{Experimental}

\subsection{Synthesis of graphene oxide}

Graphene oxide (GO) was synthesized according to the Hummers method ${ }^{[23]}$. The resulting slurry was centrifuged and washed with $2 \mathrm{~mol} / \mathrm{L} \mathrm{HCl}$ until it was $\mathrm{SO}_{4}{ }^{2-}$ free. The acid-washed GO was re-dispersed in water to form a brown dispersion. The $\mathrm{pH}$ of the dispersion was then adjusted to $\mathrm{pH}$ of 5-6 with concentrated ammonia solution to promote exfoliation of GO, and unoxidized graphite impurities were removed by centrifugation at $4000 \mathrm{rpm}$. The $\mathrm{pH}$ of the resulting supernatant 
was adjusted back to $\mathrm{pH}$ of 3 , and the supernatant was dialyzed several times in deionized (DI) water until its $\mathrm{pH}$ became close to $5^{[24]}$. The dispersion was then diluted by methanol to obtain a GO concentration of $1 \mathrm{mg} / \mathrm{mL}$.

\subsection{Synthesis of amorphous $C o-B$ alloy @GO}

All chemicals were of analytical grade, and were used without any further purification. The typical synthesis procedure of amorphous Co-B alloy@GO nanocomposite is similar to our previous work $^{[22]} \cdot \mathrm{CoCl}_{2} \cdot 6 \mathrm{H}_{2} \mathrm{O}(1.0 \mathrm{~g})$ was dissolved in distilled water $(4 \mathrm{~mL})$. Then, $0.5 \mathrm{~g}$ of $\mathrm{GO}$ was added to the resulting solution, dipped for $12 \mathrm{~h}$ (denoted as compound 1). Ethanol solution (50 mL) of sodium borohydride $(0.1 \mathrm{~mol} / \mathrm{L})$ was first prepared and adjusted to $\mathrm{pH}$ of 12 with sodium hydroxide to prevent violent hydrolysis. Compound $1(0.8675 \mathrm{~g})$ was dissolved in $5 \mathrm{~mL}$ of ethanol solution and then cooled in an ice bath. The chemical reduction reaction was carried out by adding borohydride solution dropwise into the metal salt solution while being stirred. When the addition was complete, the solution bath was continually stirred for about $1 \mathrm{~h}$ to under $\mathrm{N}_{2}$ flow to release the hydrogen. The resulting solution was centrifuged, and the precipitate was washed three times with distilled water, followed by three washing steps with ethanol. Finally, the sample was stored in absolute ethanol before use (denoted as $\mathrm{Co}-\mathrm{B} @ \mathrm{GO})$.

\subsection{Electrochemical measurements}

Pseudocapacitor electrodes were constructed by mixing the active material, carbon black and poly tetrafluoroethylene (PTFE) binder with a weight percent ratio of 75:20:5, and then the mixture was dispersed in alcohol and was pressed onto nickel foam under a pressure of $12 \mathrm{MPa}$. The geometric surface area of the prepared working electrode was $1 \mathrm{~cm}^{2}$. The electrodes were dried under vacuum at $90{ }^{\circ} \mathrm{C}$ for $1 \mathrm{~h}$ to remove the solvent. Nickel foam (1.6 mm thick, $95 \%$ purity, Goodfellow) was used as a current collector.

Cyclic voltammetry (CV), galvanostatic charge/discharge and electrochemical impedance spectroscopy (EIS) measurements were carried out with a CHI660D electrochemical workstation (ShanghaiChen Hua, Inc.). A conventional three-electrode cell was equipped with a Pt plate as the 
counter electrode and a saturated calomel electrode (SCE) electrode as the reference electrode. All experiments were carried out at room temperature and in $6 \mathrm{~mol} / \mathrm{L} \mathrm{KOH}$ solution.

\subsection{Compositional and structural characterization}

Powder X-ray diffraction (PXRD) of the samples was performed on a D/Max-RB diffractometer with $\mathrm{Cu} K_{\alpha}$ radiation $(\lambda=0.154056 \mathrm{~nm})$ and a graphite monochromator at $50 \mathrm{kV}, 100 \mathrm{~mA}$. The size and morphology of the powders were observed by transmission electron microscope (TEM) on a Hitachi H-800 microscope and scanning electron microscopy (SEM) on a JEOL-6700 microscope operating at $5 \mathrm{kV}$.

\section{Results and Discussion}

\subsection{Material characterization}

X-ray diffraction (XRD) was employed to determine the structures of the studied samples. Fig. 1 shows that the diffraction peaks of GO appear at around $2 \theta$ of $10^{\circ}$ and $42^{\circ}$, corresponding to the (002) and (111) reflections of GO, respectively, which is in accord with previous reports ${ }^{[25,26]}$. (002) and (100) diffraction peaks in graphene indicate that the synthesized graphene did not perfectly exist as a single layer ${ }^{[26]}$. It can be seen that the XRD pattern of Co-B@GO does not exhibit distinct peaks, which is consistent with our previous report ${ }^{[22]}$. According to a report by Shen and Chen ${ }^{[27]}$, the results show that the average particle size of as-prepared $\mathrm{CO}-\mathrm{B}$ and $\mathrm{Co}-\mathrm{B} @ \mathrm{GO}$ is approximately 4 nm. Moreover, no conventional stacking peaks of GO at $2 \theta=10^{\circ}$ and $42^{\circ}$ were detected in $\mathrm{Co}-$ B@GO, suggesting that the graphene sheets were homogeneously dispersed onto the surface of the Co-B nanoparticles ${ }^{[28]}$.

The detailed crystal structures were further examined by scanning electron microscopy (SEM) and transmission electron microscopy (TEM). In the SEM image of GO (Fig. 2(a)), a crumpled layered structure with several stacking layers is observed. The TEM image (Fig. 2(b)) shows that the Co-B nanoparticles are spherical particles with primary diameters between 3 and $5 \mathrm{~nm}$. This result is in agreement with the XRD results (Fig. 1) and a previous report ${ }^{[22]}$. The amorphous characteristics 
of the Co-B can be further confirmed by selected-area electron diffraction (SAED), which shows no dots or rings, indicating amorphous components. In addition, it clearly shows that the nanoparticles aggregated to a large extent, which is detrimental to the electrochemical properties. Fig. 2(c, d) show the TEM images of the as-prepared CO-B@GO nanocomposite. As estimated from the TEM image, the average size of the Co-B @ GO nanostructures is similar to that of $\mathrm{Co}-\mathrm{B}$. However, it also can be seen from the images (Fig. 2(c, d)) that aggregation of the Co-B nanoparticles is slight. The Co-B nanoparticles are highly dispersed on the GO and their dispersion is much better than the bare amorphous Co-B alloy. The inset in Fig. 2(c, d) shows the SAED patterns of GO and the Co-B@GO nanocomposite, respectively. To compare the SAED test results, no other diffraction points was observed in the SAED of Co-B@GO, indicating that amorphous Co-B alloy is loaded on the GO. The well-dispersed nanoparticles of amorphous Co-B alloy on the highly conductive GO will enhance both the ionic diffusion and electrical conduction and improve their electrochemical performance.

\subsection{Electrochemical characterization}

The prepared samples were evaluated as the active material in pseudocapacitors by employing cyclic voltammetry (CV) measurements. Fig. 3(a) shows the CV of prepared $\mathrm{Co}-\mathrm{B}$ and $\mathrm{Co}-\mathrm{B} @ \mathrm{GO}$ electrodes, recorded in $6 \mathrm{~mol} / \mathrm{L} \mathrm{KOH}$ electrolyte at a scan rate of $5 \mathrm{mV} \mathrm{s}^{-1}$. It is clearly seen that the Co-B@GO nanocomposites exhibit much higher current intensity than Co-B, suggesting the CoB@GO exhibits higher specific capacitance. Fig. 3(b) shows the CV curves of the Co-B@GO composite electrode (the absolute weight of the composites is $1.176 \mathrm{mg}$ ) measured at different scan rates of $5,10,20,30,40$ and $50 \mathrm{mV} \mathrm{s}^{-1}$ between -0.2 and $0.6 \mathrm{~V}$. Then, owing to the appearance of the redox peak pair, it reveals pronounced pseudocapacitive characteristics different from the nearly rectangular CV shapes for conventional EDLC. The specific capacitance of the active material can be calculated from the charge transferred through the forward and backward scans, which is equal to the area under the curves. The specific capacitance can then be calculated using the following equation $^{[29]}$ :

$$
C=\frac{1}{2 \times v \times \Delta m \times \Delta V} \int I \mathrm{~d} V
$$


where $C$ is the specific capacitance $\left(\mathrm{F} \mathrm{g}^{-1}\right), v$ is the scan rate $(\mathrm{V} / \mathrm{s}), \Delta m$ is the mass of the active material (g), $\Delta V$ is the potential window (V) and $I \mathrm{~d} V$ represents the area under $C V$ curve $(\mathrm{W})$. In Fig. 3(c), it can be clearly observed that Co-B@GO exhibits a high specific capacitance of $382 \mathrm{~F} \mathrm{~g}^{-1}$ at 5 $\mathrm{mV} \mathrm{s}^{-1}$ in $6 \mathrm{~mol} / \mathrm{L} \mathrm{KOH}$ solution, which is higher than those of bare amorphous Co-B alloy (308 F $\left.\mathrm{g}^{-1}\right)$ and GO powder $\left(\sim 200 \mathrm{~F} \mathrm{~g}^{-1}\right)$ reported previously ${ }^{[30,31]}$. However, the trends of rate capability curve of the two samples (Co-B@GO and Co-B) are almost the same except that the capacitance of Co-B@GO is higher, which is caused by two main reasons: on the one hand, the layered GO nanosheets in Co-B@GO nanocomposite can provide efficient charge transport channels and enhance the electrolytic accessibility, which improved electrical conductivity. On the other hand, the GO served as a carrier decreases the aggregation and improved utilization efficiency of Co-B nanoparticles ${ }^{[32,33]}$, leading to the composite electrode with increasing specific capacitance. It is the synergistic effect of the Go and Co-B nanoparticles, which enhances the electrical performance of nanocomposite.

The high rate discharge capability is crucial for an electrode material to be used efficiently for pseudocapacitor applications. Galvanostatic charge-discharge between -0.2 and $0.5 \mathrm{~V}$ was employed to estimate the electrochemical performance of samples as electrode materials for pseudocapacitor. In Fig. 4(a, b), the non-linear charge-discharge curves confirm the pseudocapacitive nature of the Co-B and $\mathrm{Co}-\mathrm{B} @ \mathrm{GO}$ electrodes, which results from the electrochemical adsorption/desorption or redox reaction at the electrode-electrolyte interface. The specific capacitance can be calculated from the galvanostatic charge-discharge curve according to Eq. $(2)^{[34]}$ :

$$
C=\frac{I \times \Delta t}{\Delta V \times \Delta m}
$$

where $C$ is the specific capacitance $\left(\mathrm{F} \mathrm{g}^{-1}\right), I$ is the constant current (A), $\Delta t$ is the discharge time (s), $\Delta V$ is the potential window $(\mathrm{V})$ and $\Delta m$ is mass of the electroactive material (g). In Fig. 4(a), the specific capacitance values are $460 \mathrm{~F} \mathrm{~g}^{-1}$ for the Co-B@ GO nanocomposites, $300 \mathrm{~F} \mathrm{~g}^{-1}$ for the Co-B nanoparticles. The obtained result is similar to the CV measurement, which also shows the enhanced electrochemical properties after loading the $\mathrm{Co}-\mathrm{B}$ nanoparticles on the GO. The remarkable enhancement in specific capacitance of the Co-B@GO nanocomposites can mainly be attributed to the high dispersibility of Co-B nanoparticles and high conductivity of GO. As seen in Fig. 4(b), the Co-B@GO nanocomposite has been investigated for galvanostatic charge-discharge study at current 
rates of $1,2,5$ and $10 \mathrm{~A} \mathrm{~g}^{-1}$ within the potential range of -0.2 to $0.45 \mathrm{~V}$. From Fig. 4(b), we can find that a specific capacitance of $460 \mathrm{~F} \mathrm{~g}^{-1}$ can be delivered at a current density of $1 \mathrm{~A} \mathrm{~g}^{-1}$. However, the specific capacitance of $\mathrm{Co}-\mathrm{B} @ \mathrm{GO}$ begins to drop as the current increases. This confirms that at the low currents, the ions have enough time to diffuse into the electrode material, while at high currents the ions can only partially penetrate into the electrode material due to the steric limitations ${ }^{[35]}$. It is worthwhile mentioning that the composites can still deliver a capacitance of $310 \mathrm{~F} \mathrm{~g}^{-1}$ even at a very high current density of $10 \mathrm{~A} \mathrm{~g}^{-1}$, indicating that the composite electrode had a good rate capability.

EIS analysis has been recognized as one of the principal methods to examine the fundamental behavior of electrode materials for pseudocapacitors. Nyquist plots in Fig. 5(a) are composed of an arc in the high frequency region and a nearly straight line in the low frequency region, where $Z^{\prime}$ and $-Z "$ are real and imaginary parts of the impedance, respectively. As shown in Fig. 5(a) inset, the EIS data can be fitted by an equivalent circuit. In Fig. 5(b), the high frequency intercept with the $X$ axis represents the combined resistance $\left(R_{\mathrm{S}}\right)$ or better known as equivalent series resistance (ESR) including the ionic resistance of the electrolyte, the intrinsic resistance of active material, and the contact resistance at the interface of the active material/electrolyte interface. The ESR of Co-B@GO nanocomposite electrode is $0.8359 \Omega$, which is lower than that of $\mathrm{Co}-\mathrm{B}$ electrode $(2.201 \Omega)$. This indicates that the Co-B@GO nanocomposite electrode has higher electrical conductivity than the bare $\mathrm{Co}-\mathrm{B}$ nanoparticles. The semicircle represents the charge transfer resistance $\left(R_{\mathrm{ct}}\right)$ at the electrode/electrolyte interface. The $R_{\mathrm{ct}}$ of $\mathrm{Co}-\mathrm{B}$ and Co-B@GO electrodes are about 0.01733 and $0.01817 \Omega$, respectively, which are similar to both electrodes. The straight lines in the low frequency region reflect the ion diffusion/transport from the electrolyte to electrode. The phase angles for impedance plots of both electrodes were observed to be higher than $45^{\circ}$ in the low frequencies clearly. These findings suggest that both electrodes are not controlled by a diffusion process. The cycling performance, another important index for electrode materials in pseudocapacitors, is shown from the specific capacitance as a function of cycle number based on CV measurement at a scan rate of $10 \mathrm{~m} \mathrm{~V}^{-1}$. As shown in Fig. 5(c), the specific capacitance gradually increases in the first 50 cycles, and thereafter, the capacitance begins to decrease. However, after 600 cycles, the specific capacitance becomes stable. After 1000 cycles, the capacitance is still approximately $84 \%$ of the first cycle, much higher than that of $\mathrm{Co}-\mathrm{B} / \mathrm{MCNA}(60 \% \text { retained })^{[22]}$, which suggests that the GO carrier greatly improves cycling stability compared to MCNA. 


\section{Conclusion}

In conclusion, a nanocomposite of $\mathrm{Co}-\mathrm{B} @ \mathrm{GO}$ was prepared by chemical reduction. The GO endows the nanocomposite with high-rate transport of both electrolyte ions and electrons throughout the electrode and high electrochemical utilization of $\mathrm{Co}-\mathrm{B}$ nanoparticles, resulting in the excellent electrochemical performance. The resulting Co-B@GO nanocomposite achieved a high specific capacitance of $460 \mathrm{~F} \mathrm{~g}^{-1}$ at a current rate of $1 \mathrm{~A} \mathrm{~g} \mathrm{~g}^{-1}$, which is about 1.5 -fold higher than that of the bare amorphous Co-B alloy. Remarkably, up to $67.4 \%$ of the capacitance is retained when the charge-discharge rate is increased 10 times from 1 to $10 \mathrm{~A} \mathrm{~g}^{-1}$, indicating the excellent high-rate properties of the as-synthesized Co-B @ GO nanocomposite. The retention of the specific capacitance, based on this initial value after a 1000 -cycle test, was $84 \%$, indicating high stability of the electrode for long cycle life. Thus, we believe that it is a promising way to anchor amorphous alloys onto conductive graphene sheets for producing high-performance and high stability electrode materials for energy storage device applications.

\section{Acknowledgements}

A part of this work was supported by the University of Minnesota Initiative for Renewable Energy and the Environment (IREE) and the Shanghai Municipal Education Commission (High-energy Beam Intelligent Processing and Green Manufacturing). Parts of this work were carried out in the Characterization Facility, University of Minnesota, which receives partial support from NSF through the MRSEC program.

\section{References}

[1] J.R. Miller, P. Simon, Sci. Magaz. 321 (2008) 651-652.

[2] X. Wang, C. Yan, A. Sumboja, P.S. Lee, Nano Energy 3 (2014) 119-126.

[3] Z. Li, Z. Xu, H. Wang, J. Ding, B. Zahiri, C.M. Holt, X. Tan, D. Mitlin, Energy Environ. Sci. 7 (2014) 1708-1718.

[4] H. Chen, L. Hu, M. Chen, Y. Yan, L. Wu, Adv. Funct. Mater. 24 (2014) 934-942.

[5] P.-C. Chen, G. Shen, Y. Shi, H. Chen, C. Zhou, ACS Nano 4 (2010) 4403-4411. 
[6] P. Simon, Y. Gogotsi, Nat. Mater. 7 (2008) 845-854.

[7] J.K. Chang, W.T. Tsai, J. Electrochem. Soc. 150 (2003) A1333-A1338.

[8] X. Zhang, W. Shi, J. Zhu, D.J. Kharistal, W. Zhao, B.S. Lalia, H.H. Hng, Q. Yan, ACS Nano 5 (2011) 2013-2019.

[9] W. Zhang, Y. Tan, Y. Gao, J. Wu, B. Tang, Ceram. Int. 41 (2015) 2626-2630.

[10] S. Ren, M. Wang, M. Xu, Y. Yang, C. Jia, C. Hao, J. Solid State Electrochem. 18 (2014) 909916.

[11] X. Cai, S.H. Lim, C.K. Poh, L. Lai, J. Lin, Z. Shen, J. Power Sources 275 (2015) 298-304.

[12] Y.G. Zhu, Y. Wang, Y. Shi, Z.X. Huang, L. Fu, H.Y. Yang, Adv. Energy Mater. 4 (2014) 10791098.

[13] M. Huang, C. Gu, X. Ge, X. Wang, J. Tu, J. Power Sources 259 (2014) 98-105.

[14] S. Yin, Y. Zhang, J. Kong, C. Zou, C.M. Li, X. Lu, J. Ma, F.Y.C. Boey, X. Chen, ACS Nano 5 (2011) 3831-3838.

[15] V. Sahu, S. Goel, R.K. Sharma, G. Singh, Nanoscale 7 (2015) 20642-20651.

[16] Y. Zhang, L. Li, S. Shi, Q. Xiong, X. Zhao, X. Wang, C. Gu, J. Tu, J. Power Sources 256 (2014) 200-205.

[17] M. Jing, Y. Yang, Y. Zhu, H. Hou, Z. Wu, X. Ji, Electrochim. Acta 141 (2014) 234-240.

[18] L.Q. Mai, F. Yang, Y.L. Zhao, X. Xu, L. Xu, Y.Z. Luo, Nat. Commun. 2 (2011) 381.

[19] X. Tian, M. Shi, X. Xu, M. Yan, L. Xu, A. Minhas-Khan, C. Han, L. He, L. Mai, Adv. Mater., 27 (2015) 7476-7482.

[20] Y. Liu, Y. Wang, L. Xiao, D. Song, Y. Wang, L. Jiao, H. Yuan, Electrochim. Acta 53 (2008) 2265-2271.

[21] Y. Wang, X. Ai, Y. Cao, H. Yang, Electrochem. Commun. 6 (2004) 780-784.

[22] W. Zhang, Y. Tan, Y. Gao, J. Wu, B. Tang, J. Solid State Electrochem. (2014) 1-6.

[23] W.S. Hummers Jr, R.E. Offeman, J. Am. Chem. Soc. 80 (1958) 1339-1339.

[24] Y. Qian, I.M. Ismail, A. Stein, Carbon 68 (2014) 221-231.

[25] K.R. Lee, K.U. Lee, J.W. Lee, B.T. Ahn, S.I. Woo, Electrochem. Commun., 12 (2010) 10521055 .

[26] B. Zhao, P. Liu, Y. Jiang, D. Pan, H. Tao, J. Song, T. Fang, W. Xu, J. Power Sources 198 (2012) 423-427. 
[27] J.H. Shen, Y.W. Chen, J. Mol. Catal. A-Chem. 273 (2007) 265-276.

[28] S. Yang, X. Feng, S. Ivanovici, K. Müllen, Angew. Chem. Int. Edit. 49 (2010) 8408-8411.

[29] M. Bode, C. Cachet, S. Bach, J.P. Pereira-Ramos, J. Ginoux, L. Yu, J. Electrochem. Soc. 144 (1997) 792-801.

[30] U. Khan, I. O’Connor, Y.K. Gun'ko, J.N. Coleman, Carbon 48 (2010) 2825-2830.

[31] Y. Zhu, S. Murali, M.D. Stoller, A. Velamakanni, R.D. Piner, R.S. Ruoff, Carbon 48 (2010) 2118-2122.

[32] Y. Cheng, S. Lu, H. Zhang, C.V. Varanasi, J. Liu, Nano Lett. 12 (2012) 4206-4211.

[33] H. Gao, F. Xiao, C.B. Ching, H. Duan, ACS Appl. Mater. Interfaces 4 (2012) 7020-7026.

[34] B. Hu, X. Qin, A.M. Asiri, K.A. Alamry, A.O. Al-Youbi, X. Sun, Electrochim. Acta 107 (2013) $339-342$.

[35] W. Xing, C. Huang, S. Zhuo, X. Yuan, G. Wang, D. Hulicova-Jurcakova, Z. Yan, G. Lu, Carbon 47 (2009) 1715-1722. 


\section{Figure captions}

Fig. 1. XRD patterns of CO-B@GO and GO.

Fig. 2. SEM micrograph of GO (a), TEM micrographs of Co-B (b) and CO-B@GO (c, d). The insets in Fig. 2(c, d) shows the SAED patterns of GO and the Co-B@GO nanocomposite, respectively.

Fig. 3. (a) Cyclic voltammetry curves of $\mathrm{Co}-\mathrm{B}$ and $\mathrm{Co}-\mathrm{B} @ \mathrm{GO}$ at a scanning rate of $5 \mathrm{mV} \mathrm{s}^{-1}$. (b) Cyclic voltammetry curves of $\mathrm{Co}-\mathrm{B} @ \mathrm{GO}$ electrode at different scan rates. (c) The specific capacitance of $\mathrm{Co}-\mathrm{B}$ and $\mathrm{Co}-\mathrm{B} @ \mathrm{GO}$ at different scan rates.

Fig. 4. (a) The charge-discharge curves of Co-B and Co-B@ GO at a current density of $1 \mathrm{~A} \mathrm{~g}^{-1}$; (b) the charge-discharge curves of Co-B@GO at different current densities; (c) the specific capacitance of Co-B@GO at various discharge current densities.

Fig. 5. (a) Impedance spectra of $\mathrm{Co}-\mathrm{B}$ and $\mathrm{Co}-\mathrm{B} @ \mathrm{GO}$ (inset is the equivalent circuit), (b) the magnification of the Nyquist curves, (c) cycling performance of $\mathrm{Co}-\mathrm{B} @ \mathrm{GO}$ at a scan rate of $10 \mathrm{~m}$ $\mathrm{V}^{-1} . Z$ is real impedance and $Z^{\prime \prime}$ is imaginary impedance. 
Figure List

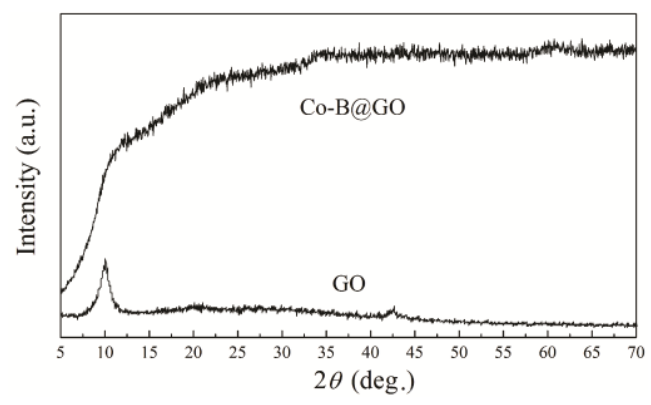

Fig. 1

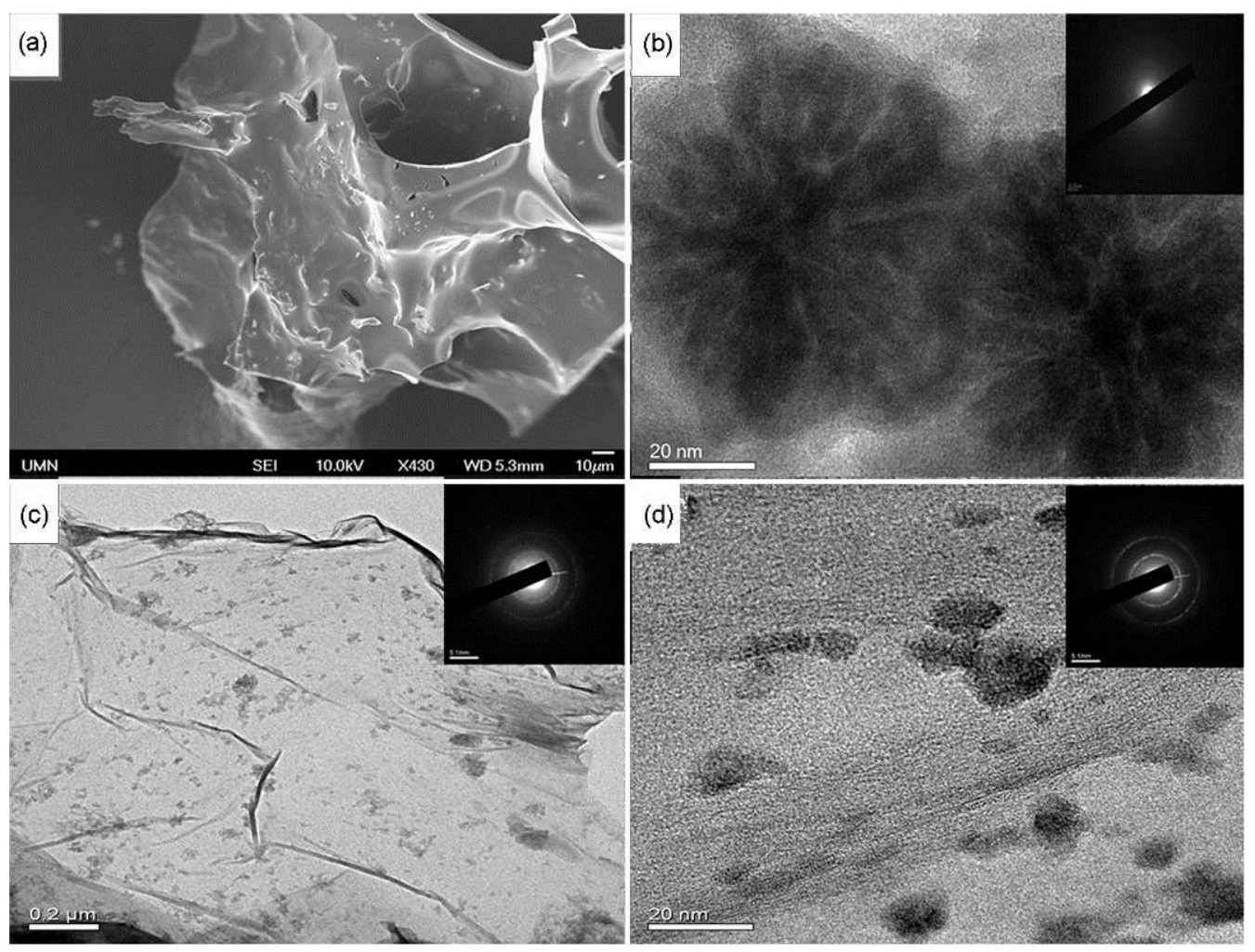

Fig. 2 

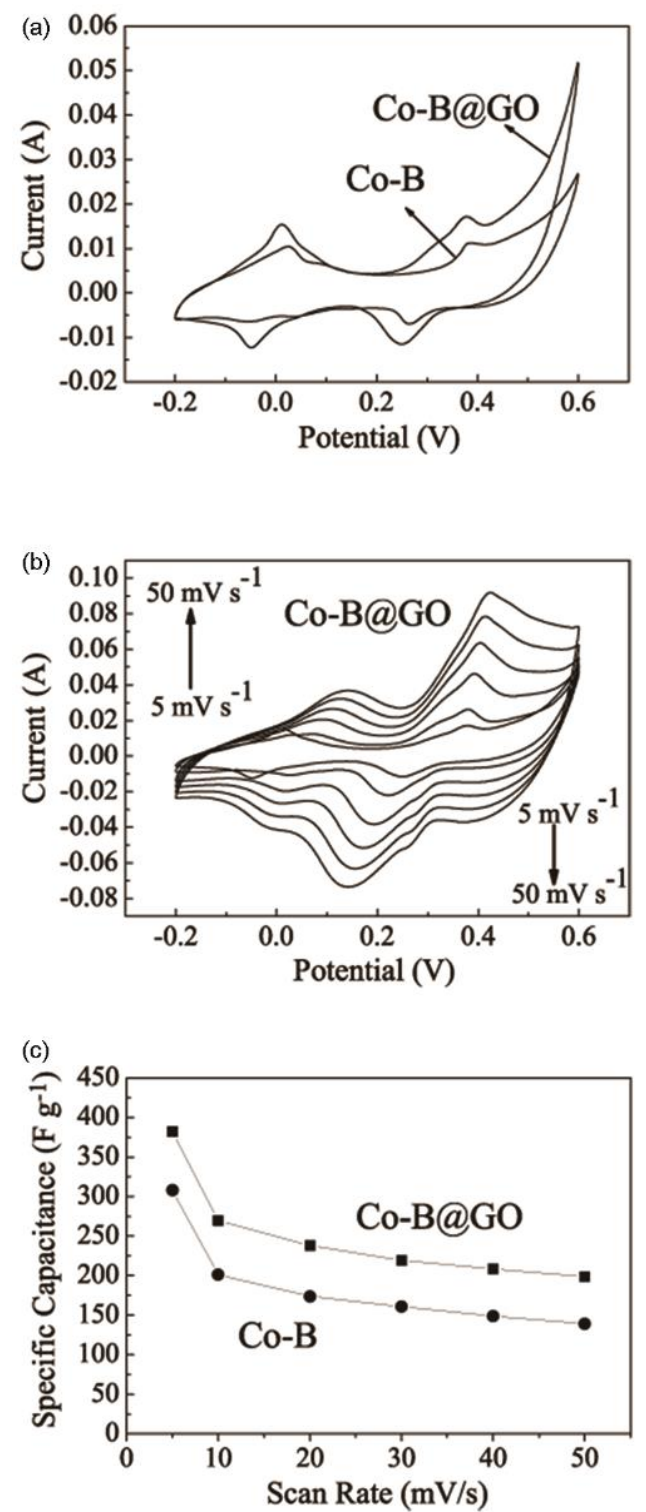

Fig. 3 

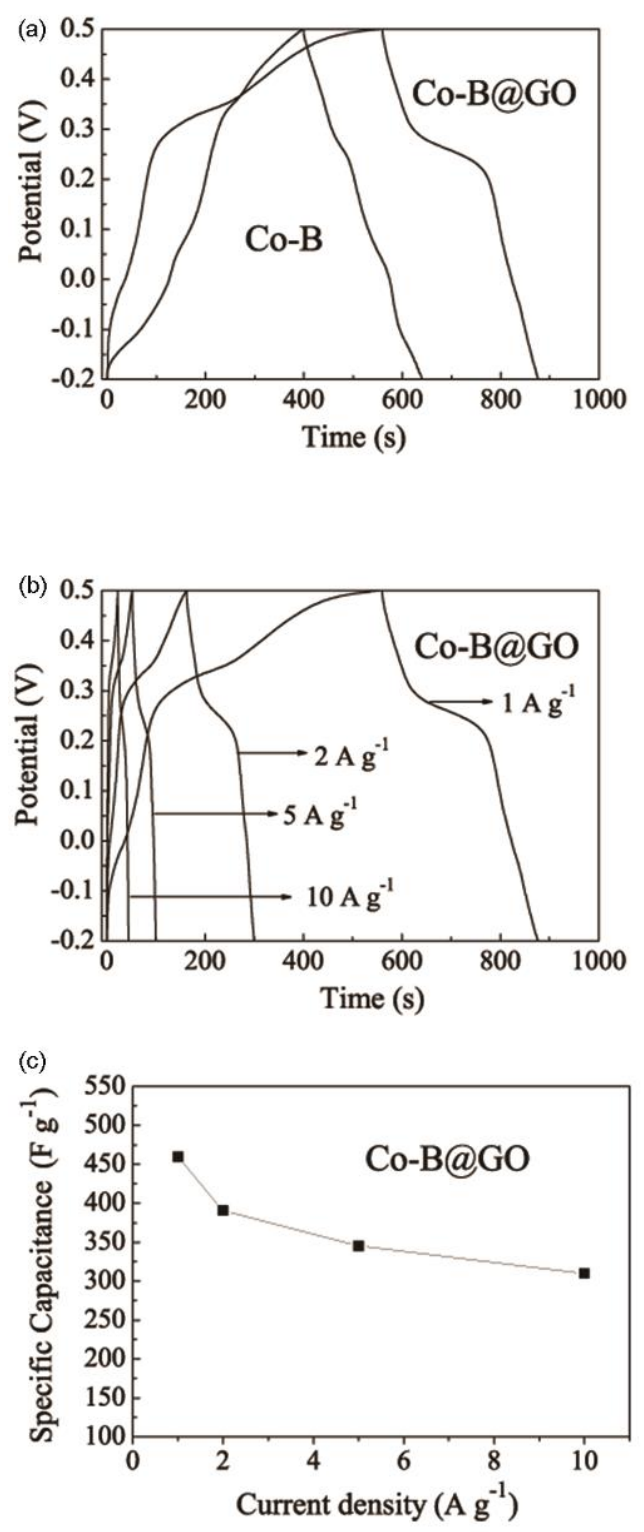

Fig. 4 

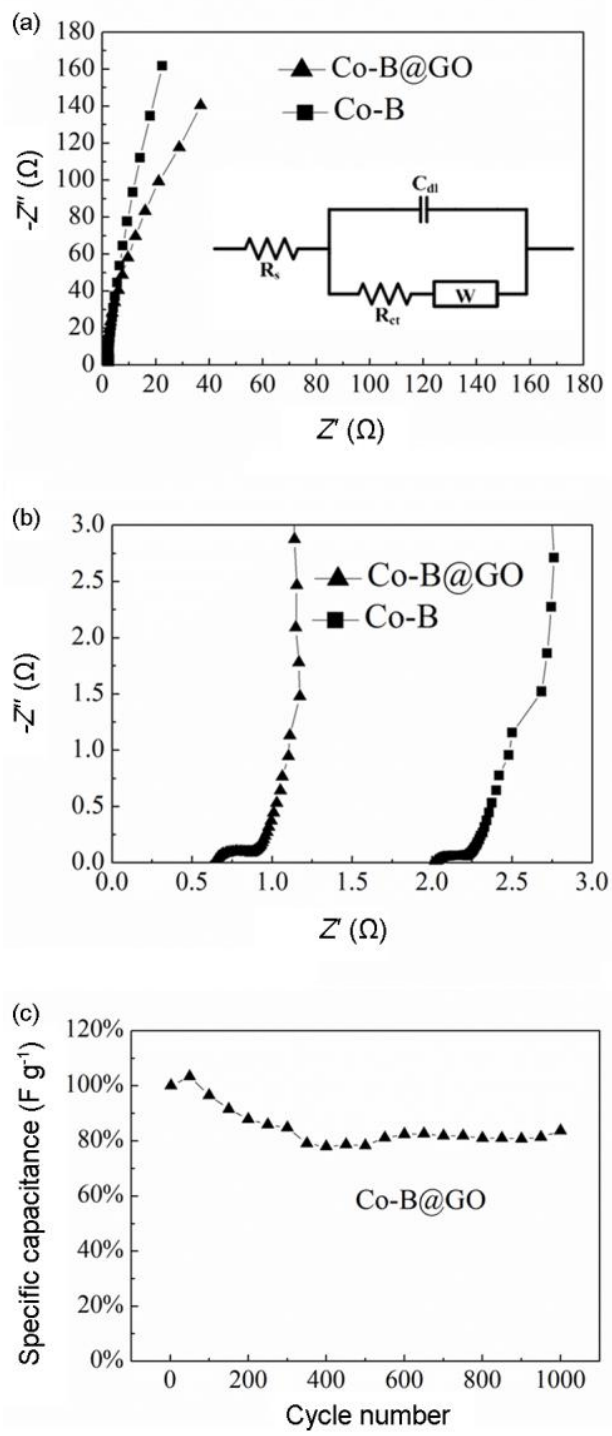

Fig. 5 